Bull. Korean Math. Soc. 51 (2014), No. 3, pp. 667-680

http://dx.doi.org/10.4134/BKMS.2014.51.3.667

\title{
ON I-SCATTERED SPACES
}

\author{
ZhaOWEn Li AND Shizhan LU
}

\begin{abstract}
In this paper, $\mathcal{I}$-scattered spaces are introduced, and their characterizations and properties are given. We prove that $(X, \tau)$ is scattered if and only if $(X, \tau, \mathcal{I})$ is $\mathcal{I}$-scattered for any ideal $\mathcal{I}$ on $X$.
\end{abstract}

\section{Introduction}

Ideals on topological spaces were studied by Kuratowski [17] and Vaidyanathaswamy [22]. Their applications have been investigated intensively (see $[4,7$, $8,13,16,18,19,21])$.

A topological space $X$ is said to be scattered if every nonempty subspace contains an isolated point. In this case we say that $X$ has a scattered topology. Every ordinal space is scattered. Scattered spaces are a class of important topological spaces. They have been researched deeply (see $[1,2,3,9,12,14$, $15])$.

The aim of this paper is to investigate scatteredness on ideal topological spaces. We introduce the concept of $\mathcal{I}$-scattered spaces, and give their characterizations and properties.

\section{Preliminaries}

Let $X$ be a nonempty set, let $2^{X}$ be the power set of $X$ and let $\mathcal{I} \subset 2^{X}$. $\mathcal{I}$ is called an ideal on $X$ if it satisfies the following conditions:

(1) If $A \in \mathcal{I}$ and $B \subset A$, then $B \in \mathcal{I}$;

(2) If $A, B \in \mathcal{I}$, then $A \cup B \in \mathcal{I}$.

If $\tau$ is a topology on $X$ and $\mathcal{I}$ is an ideal on $X$, then $(X, \tau, \mathcal{I})$ is called an ideal topological space or simply an ideal space.

Received January 22, 2013.

2010 Mathematics Subject Classification. 03E10, 54A10, 54G12.

Key words and phrases. ideals, scattered spaces, $\mathcal{I}$-scattered spaces, *-isolated points, *-derived sets, *-dense sets, *-closed mappings, topological sums.

This work is supported by the National Natural Science Foundation of China (No. 11061004), the Natural Science Foundation of Guangxi (No. 2013GXNSFBA019016) and Guangxi University Science and Technology Research Project (No. 2013ZD020, 2013ZD061). 
Let $(X, \tau, \mathcal{I})$ be an ideal space. An operator $(\cdot)^{*}: 2^{X} \longrightarrow 2^{X}$, called a local function with respect to $\tau$ and $\mathcal{I}$ [17], is defined as follows: for any $A \subset X$,

$$
A^{*}(\mathcal{I}, \tau)=\{x \in X: U \cap A \notin \mathcal{I} \text { for every } U \in \tau(x)\}
$$

where $\tau(x)=\{U \in \tau: x \in U\}$.

An operator $c l^{*}(\cdot): 2^{X} \longrightarrow 2^{X}$ is defined as follows: for any $A \subset X$,

$$
c l^{*}(A)(\mathcal{I}, \tau)=A \cup A^{*}(\mathcal{I}, \tau) .
$$

Because $c l^{*}(\cdot)$ is a Kuratowski closure operator, thus $c l^{*}(\cdot)$ generates a topology $\tau^{*}(\mathcal{I}, \tau)$, called $*$-topology on $X$. It is easy to prove that $\tau^{*}(\mathcal{I}, \tau) \supset \tau$.

When there is no chance for confusion, we will simply write $\tau^{*}$ for $\tau^{*}(\mathcal{I}, \tau)$, $A^{*}$ for $A^{*}(\mathcal{I}, \tau), c^{*} A$ for $c l^{*}(A)(\mathcal{I}, \tau)$ and $i^{*} A$ for $i n t^{*}(A)(\mathcal{I}, \tau)$, where

$$
\operatorname{int}^{*}(A)(\mathcal{I}, \tau)=X-c l^{*}(X-A)(\mathcal{I}, \tau) .
$$

$A$ is called $*$-closed [13] if $c^{*} A=A$, and $A$ is called $*$-open (i.e., $A \in \tau^{*}$ ) if $X-A$ is $*$-closed. Obviously, $A$ is $*$-open if and only if $i^{*} A=A$.

Throughout this paper, $N$ denotes the set of all natural numbers, spaces always mean topological spaces or ideal topological spaces on which no separation axiom is assumed and mappings are onto. Sometimes, $(X, \tau)$ and $(X, \tau, \mathcal{I})$ are simply written by $X . \tau^{\prime}$ (resp. $\tau^{* \prime}$ ) denotes the family of all closed (resp. *-closed) subsets of $X$. If $\mathcal{U} \subset 2^{X}, A \subset X$ and $x \in X$, then $\mathcal{U}_{A}$ denotes $\{U \cap A: U \in \mathcal{U}\}$ and $\mathcal{U}(x)$ denotes $\{U \in \mathcal{U}: x \in U\}$, the closure of $A$ and the interior of $A$ in $X$ denote, respectively, by $c A$ and $i A$, and we have

$$
i A \subset i^{*} A \subset A \subset c^{*} A \subset c A .
$$

Lemma $2.1([10])$. Let $(X, \tau, \mathcal{I})$ be an ideal space and let $A \subset X$. If $U \in \tau$, then $U \cap c^{*} A \subset c^{*}(U \cap A)$.

Proposition $2.2([20])$. Let $(X, \tau, \mathcal{I})$ be an ideal space and let $Y \subset X$. Then $\left(Y, \tau_{Y}, \mathcal{I}_{Y}\right)$ is an ideal space, where $\mathcal{I}_{Y}=\{I \cap Y: I \in \mathcal{I}\}=\{I \in \mathcal{I}: I \subset Y\}$.

Lemma $2.3([6])$. Let $(X, \tau, \mathcal{I})$ be an ideal space and let $A \subset Y \subset X$. Then $A^{*}\left(\mathcal{I}_{Y}, \tau_{Y}\right)=A^{*}(\mathcal{I}, \tau) \cap Y$.

If $(X, \tau)$ (resp. $(X, \tau, \mathcal{I}))$ is a space and $A \subset Y \subset X$, then the closure of $A$ and the interior of $A$ in the subspace $\left(Y, \tau_{Y}\right)$ (resp. $\left.\left(Y, \tau_{Y}, \mathcal{I}_{Y}\right)\right)$ are denoted by $c_{Y} A$ and $i_{Y} A$ (resp. $c_{Y}^{*} A$ and $i_{Y}^{*} A$ ), respectively.

Lemma $2.4([10])$. Let $(X, \tau, \mathcal{I})$ be an ideal space and let $A \subset Y \subset X$. Then $c_{Y}^{*}(A)=c^{*} A \cap Y$.

\section{3. *-isolated points and $*$-derived sets}

Let $(X, \tau)$ be a space and let $x \in A \subset X . x$ is called an accumulation point of $A$ in $X$ if $U \cap(A-\{x\}) \neq \emptyset$ for any $U \in \tau(x)$. The derived set of $A$ in $X$, denoted by $d(A)$, is the set of all accumulation points of $A$ in $X . x$ is called an 
isolated point of $A$ in $X$ if there exists $U \in \tau(x)$ such that $U \cap A=\{x\}$. We denote the set of all isolated points of $A$ in $X$ by $I(A)$. It is well known that

$$
I(A)=A-d(A) \text { and } c A=d(A) \cup A .
$$

Now, we introduce the concepts of $*$-isolated points and $*$-derived sets in an ideal space.

Definition 3.1. Let $(X, \tau, \mathcal{I})$ be an ideal space and let $x \in A \subset X$.

(1) $x$ is called a $*$-isolated point of $A$ in $X$ if there exists $U \in \tau^{*}(x)$ such that $U \cap A=\{x\}$.

(2) $x$ is called a $*$-accumulation point of $A$ in $X$ if $U \cap(A-\{x\}) \neq \emptyset$ for any $U \in \tau^{*}(x)$.

The set of all $*$-isolated points of $A$ in $X$ is denoted by $I^{*}(A)(\mathcal{I}, \tau)$ or $I^{*}(A)$. The set of all *-accumulation points of $A$ in $X$ is denoted by $d^{*}(A)(\mathcal{I}, \tau)$ or $d^{*}(A)$, which is called the $*$-derived set of $A$ in $X$.

Proposition 3.2. Let $(X, \tau, \mathcal{I})$ be an ideal space. Then for $A, B \subset X$,

(1) $I^{*}(A)=A-d^{*}(A)$.

(2) $I(A) \subset I^{*}(A) \subset A$.

(3) a) $A=I^{*}(A) \cup\left(d^{*}(A) \cap A\right)$; b) $d^{*}(A) \cap A=A-I^{*}(A)$.

(4) If $A \in \tau^{*}-\{\emptyset\}$ and $A \subset B$, then $I^{*}(A) \subset I^{*}(B)$.

(5) a) $I^{*}(A) \cap I^{*}(B) \subset I^{*}(A \cap B)$; b) $I^{*}(A \cup B) \subset I^{*}(A) \cup I^{*}(B)$.

Proof. (1) Let $x \in I^{*}(A)$. Then $U \cap A=\{x\}$ for some $U \in \tau^{*}(x)$. This implies $U \cap(A-\{x\})=\emptyset$. Then $x \notin d^{*}(A)$. Thus $x \in A-d^{*}(A)$ and so $I^{*}(A) \subset A-d^{*}(A)$. Conversely, let $x \in A-d^{*}(A)$. Since $x \notin d^{*}(A)$, we have $U \cap(A-\{x\})=\emptyset$ for some $U \in \tau^{*}(x)$. Note that $U \cap A=\{x\}$. Then $x \in I^{*}(A)$ and so $I^{*}(A) \supset A-d^{*}(A)$. Hence $I^{*}(A)=A-d^{*}(A)$.

(2) This is obvious.

(3) a) For any $x \in A$ and $U \in \tau^{*}(x), U \cap A=\{x\}$ or $U \cap\{A-\{x\}\} \neq \emptyset$, then $x \in I^{*}(A) \cup d^{*}(A)$ and $A \subset I^{*}(A) \cup d^{*}(A)$. Thus $A \subset\left(I^{*}(A) \cup d^{*}(A)\right) \cap A=$ $I^{*}(A) \cup\left(d^{*}(A) \cap A\right)$. And $A \supset\left(I^{*}(A) \cup d^{*}(A)\right) \cap A$. Hence $A=I^{*}(A) \cup\left(d^{*}(A) \cap\right.$ $A)$; b) This holds by a).

(4) Let $x \in I^{*}(A)$. Then $U \cap A=\{x\}$ for some $U \in \tau^{*}(x)$. Since $A \in \tau^{*}-\{\emptyset\}$, $U \cap A \in \tau^{*}-\{\emptyset\}$. Note that $(U \cap A) \cap B=\{x\}$. Then $x \in I^{*}(B)$. Thus $I^{*}(A) \subset I^{*}(B)$.

(5) This is obvious.

Proposition 3.3. Let $(X, \tau, \mathcal{I})$ and $(X, \tau, \mathcal{J})$ be two ideal spaces with $\mathcal{I} \subset \mathcal{J}$. Then for $A \subset X, I^{*}(A)(\mathcal{I}, \tau) \subset I^{*}(A)(\mathcal{J}, \tau)$.

Proof. Let $x \in I^{*}(A)(\mathcal{I}, \tau)$. Then $U \cap A=\{x\}$ for some $U \in \tau^{*}(x)(\mathcal{I}, \tau)$. It is clear that $\mathcal{I} \subset \mathcal{J}$ implies $\tau^{*}(\mathcal{I}, \tau) \subset \tau^{*}(\mathcal{J}, \tau)$. So $U \in \tau^{*}(x)(\mathcal{J}, \tau)$ and thus $x \in I^{*}(A)(\mathcal{J}, \tau)$. Hence $I^{*}(A)(\mathcal{I}, \tau) \subset I^{*}(A)(\mathcal{J}, \tau)$.

Proposition 3.4. Let $(X, \tau, \mathcal{I})$ and $(X, \sigma, \mathcal{I})$ be two ideal spaces with $\tau \subset \sigma$. Then for $A \subset X, I^{*}(A)(\mathcal{I}, \tau) \subset I^{*}(A)(\mathcal{I}, \sigma)$. 
Proof. Clearly, $\tau \subset \sigma$ implies $\tau^{*}(\mathcal{I}, \tau) \subset \sigma^{*}(\mathcal{I}, \sigma)$. Similar to the proof of Proposition 3.3, we have $I^{*}(A)(\mathcal{I}, \tau) \subset I^{*}(A)(\mathcal{I}, \sigma)$.

\section{4. $\mathcal{I}$-scattered spaces}

In this section we introduce the concept of $\mathcal{I}$-scattered spaces and give their characterizations.

\subsection{The concept of $\mathcal{I}$-scattered spaces}

Recall that a topological space $(X, \tau)$ is called scattered if every nonempty subset has its isolated points.

Below, we introduce the concept of $\mathcal{I}$-scattered spaces.

Definition 4.1. Let $(X, \tau, \mathcal{I})$ be an ideal space. $X$ is called $\mathcal{I}$-scattered if $I^{*}(A) \neq \emptyset$ for any $A \in 2^{X}-\{\emptyset\}$.

Example 4.2. Let $X=N, \tau=\{\emptyset,\{1\}\} \cup\left\{A \in 2^{N}: A \supset\{2,3\}\right\}$ and $\mathcal{I}=2^{N-\{1\}}$. Obviously, $(X, \tau, \mathcal{I})$ is an ideal space.

Let $Y=\{2,3\}$. For any $U \in \tau(2), U \cap Y \supset\{2,3\}$. Then $2 \notin I(Y)$. Similarly, $3 \notin I(Y)$. Note that $I(Y) \subset Y$. Then $I(Y)=\emptyset$. Hence $(X, \tau)$ is not scattered.

Claim. $\tau^{*}=2^{N}$.

$\{1\} \in \tau \subset \tau^{*}$.

Put $A=X-\{2\}$. Since $\{2,3\} \in \tau(2)$ and $\{2,3\} \cap A=\{3\} \in \mathcal{I}, 2 \notin A^{*}$. Then $c^{*} A=A$. Thus $\{2\} \in \tau^{*}$.

Put $B=X-\{3\}$. Since $\{2,3\} \in \tau(3)$ and $\{2,3\} \cap B=\{2\} \in \mathcal{I}, 3 \notin B^{*}$. Then $c^{*} B=B$. Thus $\{3\} \in \tau^{*}$.

Put $C_{n}=X-\{n\}$ with $n \neq 1,2,3$. Since $\{2,3, n\} \in \tau(n)$ and $\{2,3, n\} \cap C_{n}=$ $\{2,3\} \in \mathcal{I}, n \notin C_{n}^{*}$. Then $c^{*} C_{n}=C_{n}$. Thus $n \in \tau^{*}$.

Hence $\{n\} \in \tau^{*}$ for any $n \in N$. So $\tau^{*}=2^{N}$.

Let $Y \in 2^{X}-\{\emptyset\}$. For each $y \in Y$, by Claim, $\{y\} \in \tau^{*}(y)$. Since $\{y\} \cap Y=$ $\{y\}, y \in I^{*}(Y)$. Then $I^{*}(Y) \supset Y$. Note that $I^{*}(Y) \subset Y$. Then $I^{*}(Y)=Y \neq \emptyset$. Hence $(X, \tau, \mathcal{I})$ is $\mathcal{I}$-scattered.

\subsection{Characterizations of $\mathcal{I}$-scattered spaces}

Definition $4.3([11])$. Let $(X, \tau, \mathcal{I})$ be an ideal space.

(1) $A \subset X$ is called $*$-dense in $X$ if $c^{*} A=X$.

(2) $A \subset X$ is called $\mathcal{I}$-dense in $X$ if $A^{*}=X$.

Let $(X, \tau, \mathcal{I})$ be an ideal space. The family of all $*$-dense subsets of $X$ is denoted by $\mathcal{D}^{*}$. For the subspace $\left(Y, \tau_{Y}, \mathcal{I}_{Y}\right)$, the family of all $*$-dense subsets of $Y$ is denoted by $\mathcal{D}^{*}(Y)$, i.e., $\mathcal{D}^{*}(Y)=\left\{A \subset Y: c_{Y}^{*} A=Y\right\}$.

Obviously, $\mathcal{D}^{*}(X)=\mathcal{D}^{*}$.

Lemma 4.4. Let $(X, \tau, \mathcal{I})$ be an ideal space. Then $A \subset X$ is $*$-dense in $X$ if and only if $U \cap A \neq \emptyset$ for any $U \in \tau^{*}-\{\emptyset\}$. 
Proof. (Necessity) Let $A$ be $*$-dense in $X$ and let $U \in \tau^{*}-\{\emptyset\}$. Pick $x \in U$. Then $x \in X=c^{*} A=A \cup A^{*}$.

Case 1. $x \in A$.

Then $x \in U \cap A$. So $U \cap A \neq \emptyset$.

Case 2. $x \in A^{*}$.

Suppose $U \cap A=\emptyset$. Since $X-U$ is $*$-closed in $X,(X-U)^{*} \subset X-U$. Then $U \subset X-(X-U)^{*}$. By $x \in U, x \notin(X-U)^{*}$. It follows that $V \cap(X-U) \in \mathcal{I}$ for some $V \in \tau(x)$. By $U \cap A=\emptyset, A \subset X-U$. This implies $V \cap A \subset V \cap(X-U)$. Then $V \cap A \in \mathcal{I}$. So $x \notin A^{*}$, a contradiction. Thus, $U \cap A \neq \emptyset$.

(Sufficiency) Suppose $c^{*} A \neq X$. Put $U=X-c^{*} A$. Then $U \in \tau^{*}-\{\emptyset\}$. But $U \cap A=\left(X-c^{*} A\right) \cap A=\emptyset$. This is a contradiction.

Theorem 4.5. Let $(X, \tau, \mathcal{I})$ be an ideal space. The following are equivalent.

(1) $(X, \tau, \mathcal{I})$ is $\mathcal{I}$-scattered;

(2) $I^{*}(Y) \in \mathcal{D}^{*}(Y)$ for any $Y \in 2^{X}-\{\emptyset\}$;

(3) For any $Y \in 2^{X}-\{\emptyset\}, D \in \mathcal{D}^{*}(Y)$ if and only if $D \supset I^{*}(Y)$;

(4) $d^{*}(Y)=d^{*}\left(I^{*}(Y)\right)$ for any $Y \in 2^{X}-\{\emptyset\}$;

(5) If $Y \in \tau^{* \prime}-\{\emptyset\}$, then $I^{*}(Y) \neq \emptyset$.

Proof. (1) $\Rightarrow(2)$ Let $V \in \tau_{Y}^{*}-\{\emptyset\}$. Then $V=W \cap Y$ for some $W \in \tau^{*}$. Since $(X, \tau, \mathcal{I})$ is $\mathcal{I}$-scattered, $I^{*}(V) \neq \emptyset$. Pick $x \in I^{*}(V)$. Then $U \cap V=\{x\}$ for some $U \in \tau^{*}(x)$. So $(U \cap W) \cap Y=U \cap(W \cap Y)=U \cap V=\{x\}$. Note that $U \cap W \in \tau^{*}(x)$. This implies $x \in I^{*}(Y)$. Then $x \in V \cap I^{*}(Y)$ and so $V \cap I^{*}(Y) \neq \emptyset$. By Lemma 4.4, $c_{Y}^{*} I^{*}(Y)=Y$. Thus $I^{*}(Y) \in \mathcal{D}^{*}(Y)$.

$(2) \Rightarrow(3)$ Let $D \supset I^{*}(Y)$. By $(2), Y=c_{Y}^{*} I^{*}(Y) \subset c_{Y}^{*} D$. Thus $D \in \mathcal{D}^{*}(Y)$. Conversely, suppose $I^{*}(Y) \not \subset D$ for some $D \in \mathcal{D}^{*}(Y)$. Then $I^{*}(Y)-D \neq \emptyset$. Pick $x \in I^{*}(Y)-D$. Then $U \cap Y=\{x\}$ for some $U \in \tau^{*}(x)$. Note that $U \cap Y \in \tau_{Y}^{*}(x)$ and $D \in \mathcal{D}^{*}(Y)$. By Lemma 4.4, $D \cap(U \cap Y) \neq \emptyset$. But $D \cap(U \cap Y)=D \cap\{x\}=\emptyset$, a contradiction.

$(3) \Rightarrow(2)$ is obvious.

$(3) \Rightarrow(4)$ Since $Y \supset I^{*}(Y)$, we have $d^{*}(Y) \supset d^{*}\left(I^{*}(Y)\right)$. It suffices to show that $d^{*}(Y) \subset d^{*}\left(I^{*}(Y)\right)$.

Suppose $d^{*}(Y) \not \subset d^{*}\left(I^{*}(Y)\right)$. Then $d^{*}(Y)-d^{*}\left(I^{*}(Y)\right) \neq \emptyset$. Pick $x \in$ $d^{*}(Y)-d^{*}\left(I^{*}(Y)\right)$. By Proposition 3.2(1), $I^{*}(Y)=Y-d^{*}(Y)$. Then $x \notin I^{*}(Y)$. $x \notin d^{*}\left(I^{*}(Y)\right)$ implies $U \cap\left(I^{*}(Y)-\{x\}\right)=\emptyset$ for some $U \in \tau^{*}(x)$. Note that $x \notin I^{*}(Y)$. Then $(U \cap Y) \cap I^{*}(Y)=U \cap I^{*}(Y)=\emptyset$ with $U \cap Y \in \tau_{Y}^{*}$.

By $(3), I^{*}(Y) \in \mathcal{D}^{*}(Y)$. Then $V \cap I^{*}(Y) \neq \emptyset$ for any $V \in \tau_{Y}^{*}$. This is a contradiction.

Hence $d^{*}(Y)=d^{*}\left(Y-d^{*}(Y)\right)=d^{*}\left(I^{*}(Y)\right)$.

$(4) \Rightarrow(1)$ Suppose $I^{*}(Y)=\emptyset$ for some $Y \in 2^{X}-\{\emptyset\}$. By $(4), d^{*}(Y)=$ $d^{*}\left(I^{*}(Y)\right)=d^{*}(\emptyset)=\emptyset$. By Proposition 3.2(3), $Y=I^{*}(Y) \cup\left(d^{*}(Y) \cap Y\right)=\emptyset$, a contradiction.

$(1) \Rightarrow(5)$ is obvious.

$(5) \Rightarrow(1)$ Let $Y \in 2^{X}-\{\emptyset\}$. Since $c^{*} Y \in \tau^{* \prime}-\{\emptyset\}$, by $(5), I^{*}\left(c^{*} Y\right) \neq \emptyset$. Pick $x \in I^{*}\left(c^{*} Y\right)$. Then $U \cap c^{*} Y=\{x\}$ for some $U \in \tau^{*}(x)$. 
Suppose $U \cap Y=\emptyset$. We have $X-U \supset Y$. Then $X-U \supset c^{*} Y$. So $U \cap c^{*} Y=\emptyset$. This is a contradiction. Thus $U \cap Y \neq \emptyset$.

Since $U \cap Y \subset U \cap c^{*} Y=\{x\}$, we have $U \cap Y=\{x\}$. So $x \in I^{*}(Y)$. This implies $I^{*}(Y) \neq \emptyset$. Hence $X$ is $\mathcal{I}$-scattered.

Definition 4.6. Let $(X, \tau, \mathcal{I})$ be an ideal space. Put $X^{0}=X$ and

$$
X^{1}=\{x \in X: x \text { is not } * \text {-isolated in } X\} .
$$

Let $\alpha$ be any ordinal number. If $X^{\beta}$ is already defined for all ordinal $\beta<\alpha$, then we put

$$
X^{\alpha}=\left\{\begin{array}{l}
\left(X^{\beta}\right)^{1}, \quad \text { if } \alpha=\beta+1 \text { and } \beta \text { is an ordinal number, } \\
\bigcap_{\beta<\alpha} X^{\beta}, \text { if } \alpha \text { is a limit ordinal number. }
\end{array}\right.
$$

Remark 4.7. (1) $X^{1}=X-I^{*}(X)=X \cap d^{*}(X)$.

(2) $X^{\alpha} \supset X^{\beta}$ whenever $\alpha \leq \beta$.

(3) $X^{\alpha}=X^{\alpha-1}-I^{*}\left(X^{\alpha-1}\right)=X^{\alpha-1} \cap d^{*}\left(X^{\alpha-1}\right)$ for any successor ordinal number $\alpha$.

(4) If $\alpha$ is a successor ordinal number and $X^{\alpha}=\emptyset$, then $X=\bigcup_{\beta \leq \alpha-1} I^{*}\left(X^{\beta}\right)$.

Lemma 4.8. $X^{\delta}=X^{\delta+1}$ for some ordinal number $\delta$.

Proof. Put $|X|=k$.

Case 1. There are $\alpha, \beta<k+1(\alpha \neq \beta)$ such that $X^{\alpha}=X^{\beta}$.

We may suppose $\alpha<\beta$. By Remark 4.7, $X^{\beta}=X^{\alpha} \supset X^{\alpha+1} \supset \cdots \supset X^{\beta}$. Then $X^{\alpha}=X^{\alpha+1}$. Pick $\delta=\alpha$. Then $X^{\delta}=X^{\delta+1}$.

Case 2. $X^{\alpha} \neq X^{\beta}$ for any $\alpha, \beta<k+1(\alpha \neq \beta)$.

By Definition 4.6, $I^{*}\left(X^{\gamma}\right) \neq \emptyset$ for every $\gamma<k+1$. Then $X^{k+1}=X^{k+2}=\emptyset$. Pick $\delta=k+1$. Then $X^{\delta}=X^{\delta+1}$.

Lemma 4.9. Let $(X, \tau, \mathcal{I})$ be an ideal space. The following properties hold.

(1) $X^{\alpha} \in \tau^{* \prime}$ for any ordinal number $\alpha$.

(2) If $Y \subset X$, then $Y^{\alpha} \subset X^{\alpha}$ for any ordinal number $\alpha$.

Proof. (1) We use induction on $\alpha$.

1) $\alpha=1$. Let $x \in I^{*}(X)$. Then $U_{x} \cap X=\{x\}$ for some $U_{x} \in \tau^{*}(x)$. This implies $\{x\}=U_{x} \in \tau^{*}$. Thus $I^{*}(X)=\bigcup_{x \in I^{*}(X)}\{x\} \in \tau^{*}$. Thus $X^{1}=$ $X-I^{*}(X) \in \tau^{* \prime}$.

2) Suppose $X^{\beta} \in \tau^{*^{\prime}}$ for any $\beta<\alpha$. We will prove $X^{\alpha} \in \tau^{* \prime}$ in the following cases.

a) $\alpha$ is a successor ordinal number.

Let $x \in I^{*}\left(X^{\alpha-1}\right)$. Then $U_{x} \cap X^{\alpha-1}=\{x\}$ for some $U_{x} \in \tau^{*}(x)$. By Remark 4.7, $X^{\alpha}=X^{\alpha-1}-I^{*}\left(X^{\alpha-1}\right)$. So

$$
X^{\alpha}=X^{\alpha-1}-\bigcup_{x \in I^{*}\left(X^{\alpha-1}\right)}\{x\}=\left(X-\bigcup_{x \in I^{*}\left(X^{\alpha-1}\right)} U_{x}\right) \cap X^{\alpha-1} .
$$


By induction hypothesis, $X^{\alpha-1} \in \tau^{* \prime}$. Thus $X^{\alpha} \in \tau^{* \prime}$.

b) $\alpha$ is a limit ordinal number.

By induction hypothesis, $X^{\beta} \in \tau^{*^{\prime}}$ for any $\beta<\alpha$. Thus $X^{\alpha}=\bigcap_{\beta<\alpha} X^{\beta} \in$ $\tau^{* \prime}$.

(2) Let $Y \subset X$. We will prove $Y^{\alpha} \subset X^{\alpha}$ for any ordinal number $\alpha$.

1) $Y^{1}=Y \cap d^{*}(Y) \subset X \cap d^{*}(X)=X^{1}$.

This shows $Y^{\alpha} \subset X^{\alpha}$ when $\alpha=1$.

2) Suppose $Y^{\beta} \subset X^{\beta}$ for any $\beta<\alpha$. We consider the following cases.

a) $\alpha$ is a successor ordinal number.

By induction hypothesis, $Y^{\alpha-1} \subset X^{\alpha-1}$. By Remark 4.7,

$$
Y^{\alpha}=Y^{\alpha-1} \cap d^{*}\left(Y^{\alpha-1}\right) \subset X^{\alpha-1} \cap d^{*}\left(X^{\alpha-1}\right)=X^{\alpha}
$$

b) $\alpha$ is a limit ordinal number.

By induction hypothesis, $Y^{\beta} \subset X^{\beta}$ for any $\beta<\alpha$. Thus

$$
Y^{\alpha}=\bigcap_{\beta<\alpha} Y^{\beta} \subset \bigcap_{\beta<\alpha} X^{\beta}=X^{\alpha} .
$$

By 1) and 2), $Y^{\alpha} \subset X^{\alpha}$.

Definition 4.10. Let $(X, \tau, \mathcal{I})$ be an ideal space.

(1) An ordinal number $\beta$ is called the derived length of $X$ if $\beta=\min \{\alpha$ : $\left.X^{\alpha}=\emptyset\right\} . \beta$ is denoted by $\delta(X)$.

(2) $X$ is called to have a derived length if there is an ordinal number $\alpha$ such that $X^{\alpha}=\emptyset$.

Theorem 4.11. Let $(X, \tau, \mathcal{I})$ be an ideal space. Then $X$ is $\mathcal{I}$-scattered if and only if $X$ has a derived length.

Proof. (Sufficiency) Suppose that $X$ is not $\mathcal{I}$-scattered. Then $I^{*}(A)=\emptyset$ for some $A \in 2^{X}-\{\emptyset\}$.

Claim. $A \subset X^{\alpha}$ for any ordinal number $\alpha$.

(1) Let $x \in A$ and $U \in \tau^{*}(x)$. Since $I^{*}(A)=\emptyset, U \cap A \neq\{x\}$. Note that $x \in U \cap A$. Then $|U \cap A| \geqslant 2$ and so $U \cap(A-\{x\}) \neq \emptyset$. Now $U \cap(X-\{x\}) \supset$ $U \cap(A-\{x\})$. Then $U \cap(X-\{x\}) \neq \emptyset$. This implies $x \in d^{*}(X) \cap X$. By Remark 4.7, $x \in X^{1}$.

Thus $A \subset X-I^{*}(X)=X^{1}$, i.e., $A \subset X^{\alpha}$ when $\alpha=1$.

(2) Suppose $A \subset X^{\beta}$ for any $\beta<\alpha$. We will prove $A \subset X^{\alpha}$ in the following cases.

a) $\alpha$ is a successor ordinal number.

Let $x \in A$ and $U \in \tau^{*}(x)$. By the proof above, $U \cap(A-\{x\}) \neq \emptyset$. By induction hypothesis, $A \subset X^{\alpha-1}$. Then $U \cap\left(X^{\alpha-1}-\{x\}\right) \neq \emptyset$. This implies $x \in d^{*}\left(X^{\alpha-1}\right) \cap X^{\alpha-1}$. By Remark 4.7, $x \in X^{\alpha}$.

Hence $A \subset X^{\alpha}$.

b) $\alpha$ is a limit ordinal number.

By induction hypothesis, $A \subset X^{\beta}$ for any $\beta<\alpha$. Then $A \subset \bigcap_{\beta<\alpha} X^{\beta}=X^{\alpha}$. 
Since $X$ has a derived length, $X^{\delta}=\emptyset$ for some ordinal number $\delta$. By Claim, $A \subset X^{\alpha}$. Then $A=\emptyset$, a contradiction.

(Necessity) Suppose that $X$ has no derived length. By Lemma $4.8, X^{\delta}=$ $X^{\delta+1}$ for some ordinal number $\delta$. By Remark 4.7, $X^{\delta+1}=X^{\delta}-I^{*}\left(X^{\delta}\right)$. Then $I^{*}\left(X^{\delta}\right)=\emptyset$. Note that $X$ has no derived length. Then $X^{\delta} \neq \emptyset$. It follows that $X$ is not $\mathcal{I}$-scattered, a contradiction.

\section{Some properties of $\mathcal{I}$-scattered spaces}

In this section we will give some properties of $\mathcal{I}$-scattered spaces.

\subsection{Simple properties of $\mathcal{I}$-scattered spaces}

Theorem 5.1. Let $(X, \tau, \mathcal{I})$ and $(X, \tau, \mathcal{J})$ be two ideal spaces.

(1) If $\mathcal{I} \subset \mathcal{J}$ and $(X, \tau, \mathcal{I})$ is $\mathcal{I}$-scattered, then $(X, \tau, \mathcal{J})$ is $\mathcal{J}$-scattered.

(2) If $\tau \subset \sigma$ and $(X, \tau, \mathcal{I})$ is $\mathcal{I}$-scattered, then $(X, \sigma, \mathcal{I})$ is $\mathcal{I}$-scattered.

Proof. These hold by Proposition 3.3 and Proposition 3.4.

Theorem 5.2. Let $(X, \tau)$ be a space. The following are equivalent.

(1) $(X, \tau)$ is scattered.

(2) $(X, \tau,\{\emptyset\})$ is $\{\emptyset\}$-scattered.

(3) $(X, \tau, \mathcal{I})$ is $\mathcal{I}$-scattered for any ideal $\mathcal{I}$ on $X$.

Proof. (1) $\Rightarrow$ (3) follows from Proposition 3.2(2).

$(3) \Rightarrow(2)$ is obvious.

$(2) \Rightarrow(1)$ Since $\tau^{*}=\tau$ whenever $\mathcal{I}=\{\emptyset\}, I(A)=I^{*}(A) \neq \emptyset$. Thus $(X, \tau)$ is scattered.

Theorem 5.3. Let $(X, \tau, \mathcal{I})$ be an ideal space and let $Y \in 2^{X}-\{\emptyset\}$. If $X$ is $\mathcal{I}$-scattered, then $\left(Y, \tau_{Y}, \mathcal{I}_{Y}\right)$ is $\mathcal{I}_{Y}$-scattered.

Proof. Let $A \in 2^{Y}-\{\emptyset\}$. Since $X$ is $\mathcal{I}$-scattered, $I^{*}(A) \neq \emptyset$. Pick $x \in I^{*}(A)$. Then $U \cap A=\{x\}$ for some $U \in \tau^{*}(x)$. Note that $U \cap Y \in \tau_{Y}^{*}(x)$ and $(U \cap Y) \cap A=(U \cap A) \cap Y=\{x\}$. Then $x \in I_{Y}^{*}(A)$ and so $I_{Y}^{*}(A) \neq \emptyset$. Hence $\left(Y, \tau_{Y}, \mathcal{I}_{Y}\right)$ is $\mathcal{I}_{Y \text {-scattered. }}$

\section{2. $\mathcal{I}$-scatteredness and topological sums}

Lemma $5.4([5])$. If every $\mathcal{I}_{\alpha}$ is an ideal on $X_{\alpha}(\alpha \in \Gamma)$, then $\left\{\bigcup_{\alpha \in \Gamma} I_{\alpha}: I_{\alpha} \in\right.$ $\left.\mathcal{I}_{\alpha}\right\}$ is an ideal of $\bigcup_{\alpha \in \Gamma} X_{\alpha}$.

Let $\left\{\left(X_{\alpha}, \tau_{\alpha}, \mathcal{I}_{\alpha}\right): \alpha \in \Gamma\right\}$ be a family of pairwise disjoint ideal spaces, i.e., $X_{\alpha} \cap X_{\beta}=\emptyset$ for $\alpha \neq \beta$.

Put

$$
X=\bigcup_{\alpha \in \Gamma} X_{\alpha}
$$

$$
\tau=\left\{A \subset X: A \cap X_{\alpha} \in \tau_{\alpha} \text { for each } \alpha \in \Gamma\right\}
$$


and

$$
\mathcal{I}=\left\{\bigcup_{\alpha \in \Gamma} I_{\alpha}: I_{\alpha} \in \mathcal{I}_{\alpha}\right\}
$$

It is easy to prove that $\tau$ is a topology on $X$ and every $X_{\alpha}$ is clopen in $X$. By Lemma 5.4, $(X, \tau, \mathcal{I})$ is an ideal space, which is said to be the sum of $\left\{\left(X_{\alpha}, \tau_{\alpha}, \mathcal{I}_{\alpha}\right): \alpha \in \Gamma\right\}$. We also denote it by $\bigoplus_{\alpha \in \Gamma} X_{\alpha}$.

Lemma 5.5. Let $(X, \tau, \mathcal{I})$ be the sum of $\left\{\left(X_{\alpha}, \tau_{\alpha}, \mathcal{I}_{\alpha}\right): \alpha \in \Gamma\right\}$ and let $A \subset X$. Then $A \in \tau^{* \prime}$ if and only if $A \cap X_{\alpha} \in \tau^{* \prime}{ }_{\alpha}^{\prime}$ for each $\alpha \in \Gamma$.

Proof. (Necessity) Suppose $A \in \tau^{* \prime}$. It suffices to show $\left(A \cap X_{\alpha}\right)^{*}\left(\mathcal{I}_{\alpha}, \tau_{\alpha}\right) \subset$ $A \cap X_{\alpha}$.

Suppose $\left(A \cap X_{\alpha}\right)^{*}\left(\mathcal{I}_{\alpha}, \tau_{\alpha}\right) \not \subset A \cap X_{\alpha}$. Then $\left(A \cap X_{\alpha}\right)^{*}\left(\mathcal{I}_{\alpha}, \tau_{\alpha}\right)-A \cap X_{\alpha} \neq \emptyset$. Pick $x_{0} \in\left(A \cap X_{\alpha}\right)^{*}\left(\mathcal{I}_{\alpha}, \tau_{\alpha}\right)-A \cap X_{\alpha}$. Since $x_{0} \in\left(A \cap X_{\alpha}\right)^{*}\left(\mathcal{I}_{\alpha}, \tau_{\alpha}\right), x_{0} \in X_{\alpha}$ and $U \cap\left(A \cap X_{\alpha}\right) \notin \mathcal{I}_{\alpha}$ for each $U \in \tau_{\alpha}\left(x_{0}\right)$. Note that $x_{0} \notin A \cap X_{\alpha}$ and $A \in \tau^{* \prime}$. Then $x_{0} \notin A=c^{*}(A)=A \cup A^{*}$ and so $x_{0} \notin A^{*}(\mathcal{I}, \tau)$. Thus $U_{0} \cap A \in \mathcal{I}$ for some $U_{0} \in \tau\left(x_{0}\right)$. This implies $U_{0} \cap A=\bigcup_{\beta \in \Gamma} I_{\beta}$ where $I_{\beta} \in \mathcal{I}_{\beta}$. Then

$$
\left(U_{0} \cap A\right) \cap X_{\alpha}=\left(\bigcup_{\beta \in \Gamma} I_{\beta}\right) \cap X_{\alpha}=\left(I_{\alpha} \cap X_{\alpha}\right) \cup\left(\bigcup_{\beta \neq \alpha}\left(I_{\beta} \cap X_{\alpha}\right)\right)=I_{\alpha} \in \mathcal{I}_{\alpha} .
$$

Since $U \cap\left(A \cap X_{\alpha}\right) \notin \mathcal{I}_{\alpha}$ for each $U \in \tau_{\alpha}\left(x_{0}\right)$, a contradiction. Hence $A \cap X_{\alpha} \in$ $\tau_{\alpha}^{* \prime}$.

(Sufficiency) Let $A \cap X_{\alpha} \in \tau^{* \prime}{ }_{\alpha}$ for each $\alpha \in \Gamma$. Now we prove $A^{*} \subset A$.

Suppose $A^{*}-A \neq \emptyset$. Pick $x_{0} \in A^{*}-A$. We have $x_{0} \notin A$ and $U \cap A \notin \mathcal{I}$ for each $U \in \tau\left(x_{0}\right)$. Since $x_{0} \in U=U \cap X=U \cap\left(\bigcup_{\alpha \in \Gamma} X_{\alpha}\right)=\bigcup_{\alpha \in \Gamma}\left(U \cap X_{\alpha}\right)$, $x_{0} \in U \cap X_{\beta} \in \tau_{\beta}$ for some $\beta \in \Gamma$. Then $U \cap X_{\beta} \in \tau_{\beta}\left(x_{0}\right)$.

Claim. $x_{0} \in\left(A \cap X_{\beta}\right)^{*}\left(\mathcal{I}_{\beta}, \tau_{\beta}\right)$.

Suppose $x_{0} \notin\left(A \cap X_{\beta}\right)^{*}\left(\mathcal{I}_{\beta}, \tau_{\beta}\right)$. Then $\left(A \cap X_{\beta}\right) \cap W \in \mathcal{I}_{\beta}$ for some $W \in$ $\tau_{\beta}\left(x_{0}\right)$. Note that $W \subset X_{\beta}$. Then $A \cap W=\left(A \cap X_{\beta}\right) \cap W$ and so $A \cap W \in \mathcal{I}_{\beta}$. Obviously, $W \in \tau\left(x_{0}\right)$. Since $\emptyset \in \mathcal{I}_{\alpha}(\alpha \in \Gamma), A \cap W \in \mathcal{I}$. Since $A \cap U \notin \mathcal{I}$ for any $U \in \tau\left(x_{0}\right)$, a contradiction. Thus $x_{0} \in\left(A \cap X_{\beta}\right)^{*}\left(\mathcal{I}_{\beta}, \tau_{\beta}\right)$.

Now $A \cap X_{\beta} \in \tau_{\beta}^{* \prime}$. By hypothesis, we have $\left(A \cap X_{\beta}\right)^{*}\left(\mathcal{I}_{\beta}, \tau_{\beta}\right) \subset A \cap X_{\beta} \subset A$. This implies $x_{0} \in A$, a contradiction.

Lemma 5.6. Let $(X, \tau, \mathcal{I})$ be the sum of $\left\{\left(X_{\alpha}, \tau_{\alpha}, \mathcal{I}_{\alpha}\right): \alpha \in \Gamma\right\}$. Then for $A \subset X, A \in \tau^{*}$ if and only if $A \cap X_{\alpha} \in \tau_{\alpha}^{*}$ for each $\alpha \in \Gamma$.

Proof. This holds by Lemma 5.5.

Theorem 5.7. Let $(X, \tau, \mathcal{I})$ be the sum of $\left\{\left(X_{\alpha}, \tau_{\alpha}, \mathcal{I}_{\alpha}\right): \alpha \in \Gamma\right\}$. Then $(X, \tau, \mathcal{I})$ is $\mathcal{I}$-scattered if and only if $\left(X_{\alpha}, \tau_{\alpha}, \mathcal{I}_{\alpha}\right)$ is $\mathcal{I}_{\alpha}$-scattered for each $\alpha \in \Gamma$.

Proof. (Sufficiency) Let $A \in 2^{X}-\{\emptyset\}$. Since $A=\bigcup_{\alpha \in \Gamma}\left(A \cap X_{\alpha}\right), A \cap X_{\beta} \neq \emptyset$ for some $\beta \in \Gamma$. By $X_{\beta}$ is $\mathcal{I}_{\beta}$-scattered, $I_{X_{\beta}}^{*}\left(A \cap X_{\beta}\right) \neq \emptyset$. Pick $x \in I_{X_{\beta}}^{*}\left(A \cap X_{\beta}\right)$. Then there exists $U \in \tau_{\beta}^{*}(x)$ such that $U \cap\left(X_{\beta} \cap A\right)=\{x\}=U \cap A$. Since

$$
U \cap X_{\alpha}= \begin{cases}U \in \tau_{\beta}^{*}, & \alpha=\beta, \\ \emptyset \in \tau_{\alpha}^{*}, & \alpha \neq \beta,\end{cases}
$$


by Lemma 5.6, $U \in \tau^{*}(x)$. This implies $x \in I^{*}(A)$. Then $I^{*}(A) \neq \emptyset$. Thus $(X, \tau, \mathcal{I})$ is $\mathcal{I}$-scattered.

(Necessity) Obviously, $\tau_{X_{\alpha}}=\tau_{\alpha}$ and $I_{X_{\alpha}}=I_{\alpha}$ for any $\alpha \in \Gamma$. By Theorem 5.3 , every $\left(X_{\alpha}, \tau_{\alpha}, \mathcal{I}_{\alpha}\right)$ is $\mathcal{I}_{\alpha}$-scattered.

\section{3. $\mathcal{I}$-scatteredness and $\mathcal{I}$-irresolvableness}

Definition $5.8([7])$. An ideal space $(X, \tau, \mathcal{I})$ is called $\mathcal{I}$-resolvable if $X$ has two disjoint $\mathcal{I}$-dense subsets. Otherwise, $X$ is called $\mathcal{I}$-irresolvable.

Theorem 5.9. Let $(X, \tau, \mathcal{I})$ be an ideal space. If $X$ is $\mathcal{I}$-scattered, then $X$ is $\mathcal{I}$-irresolvable.

Proof. For any $A, B \in 2^{X}-\{\emptyset\}$ with $A^{*}=B^{*}=X$ and $X=A \cup B$, we have $A, B \in \mathcal{D}^{*}(X)$. By Theorem 4.5, $A, B \supset I^{*}(X)$. Then $A \cap B \supset I^{*}(X)$. Since $X$ is $\mathcal{I}$-scattered, $I^{*}(X) \neq \emptyset$. So $A \cap B \neq \emptyset$. Thus, $X$ is $\mathcal{I}$-irresolvable.

Example 5.10. $\mathcal{I}$-irresolvable $\Rightarrow \mathcal{I}$-scattered.

Let $X=R, \tau=\{\emptyset, X,\{0\}\}, \mathcal{I}=\{\emptyset,\{0\}\}$. Then $(X, \tau, \mathcal{I})$ is an ideal space.

(1) Claim 1. $0 \notin A^{*}$ for any $A \in 2^{X}$.

Obviously, $\{0\} \in \tau(0)$. By $\{0\} \cap A=\emptyset \in \mathcal{I}$ or $\{0\} \cap A=\{0\} \in \mathcal{I}$, we have $0 \notin A^{*}$. Then $0 \notin A^{*}$ for any $A \in 2^{X}$.

This implies that for any $A, B \in 2^{X}-\{\emptyset\}, A^{*} \neq X$ and $B^{*} \neq X$. Thus $X$ is $\mathcal{I}$-irresolvable.

(2) Claim 2. $\{a\}^{*}=X-\{0\}$ when $a \in X-\{0\}$.

By Claim 1, $0 \notin\{a\}^{*}$. Then $\{a\}^{*} \subset X-\{0\}$. Conversely, for any $x \in$ $X-\{0\}$ and $U \in \tau(x), \tau(x)=\{X\}$, we have $U=X$. Since $U \cap\{a\}=\{a\} \notin \mathcal{I}$, $x \in\{a\}^{*}$. Then $X-\{0\} \subset\{a\}^{*}$. Thus $\{a\}^{*}=X-\{0\}$.

Claim 3. $A^{*}=X-\{0\}$ when $A \in 2^{X}-\{\emptyset,\{0\}\}$.

By Claim 1, $0 \notin A^{*}$. Then $A^{*} \subset X-\{0\}$. Conversely, by $A \in 2^{X}-\{\emptyset,\{0\}\}$, $A \cap(X-\{0\}) \neq \emptyset$. Pick $a \in A \cap(X-\{0\})$. Then $A^{*} \supset\{a\}^{*}$. By Claim 2, $\{a\}^{*}=X-\{0\}$. This implies $A^{*} \supset X-\{0\}$. Thus $A^{*}=X-\{0\}$ when $A \in 2^{X}-\{\emptyset,\{0\}\}$.

Claim 4. $\tau^{* \prime}=\{\emptyset, X,\{0\}, X-\{0\}\}$.

Obviously, $\{0\}^{*}=\emptyset$. Then $\{0\} \in \tau^{* \prime}$. Let $A \in 2^{X}-\{\emptyset, X,\{0\}, X-\{0\}\}$. By Claim 3, $A^{*}=X-\{0\}$. This implies $c^{*} A=X-\{0\} \neq A$. Then $A \notin \tau^{* \prime}$. Thus $\tau^{* \prime}=\{\emptyset, X,\{0\}, X-\{0\}\}$.

Put $B=\{1,2\}$. By Claim 4, $\tau^{*}=\{\emptyset, X,\{0\}, X-\{0\}\}$. Then $\tau^{*}(1)=$ $\{X, X-\{0\}\}$. Let $x \in X$. (a) $x \in X-B$. Then $x \notin I^{*}(B)$ since $I^{*}(B) \subset B$. (b) $x \in B$. Let $U \in \tau^{*}(x)$. Since $\tau^{*}(1)=\tau^{*}(2)=\{X, X-\{0\}\}, U \cap B=B \neq\{x\}$. Then $x \notin I^{*}(B)$. Thus, $I^{*}(B)=\emptyset$. This shows that $X$ is not $\mathcal{I}$-scattered.

\subsection{Mapping properties of $\mathcal{I}$-scattered spaces}

In this subsection we study the $*$-closed continuous images of $\mathcal{I}$-scattered spaces. 
Definition 5.11 ([8]). A mapping $f:(X, \tau, \mathcal{I}) \rightarrow(Y, \sigma, \mathcal{J})$ is called $*$-closed, if $f(A)$ is $*$-closed in $Y$ for each $*$-closed subset $A$ of $X$.

Theorem 5.12. Let $(X, \tau, \mathcal{I})$ be $\mathcal{I}$-scattered, let $(Y, \sigma, \mathcal{J})$ be an ideal space and let $f:(X, \tau, \mathcal{I}) \rightarrow(Y, \sigma, \mathcal{J})$ be $*$-closed. Suppose that $f$ satisfies the following condition $(*)$ :

The set $\left\{\beta: X^{\beta} \cap f^{-1}(y) \neq \emptyset\right\}$ contains a largest element for any $y \in Y$.

Then the following properties hold:

(i) $Y^{\alpha} \subset f\left(X^{\alpha}\right)$ for every ordinal number $\alpha$,

(ii) $\delta(Y) \leqslant \delta(X)$,

(iii) $Y$ is $\mathcal{J}$-scattered.

Proof. Since (ii) and (iii) hold by (i) and Theorem 4.11, we only need to prove (i), i.e., $Y^{\alpha} \subset f\left(X^{\alpha}\right)$ for any ordinal number $\alpha$.

We use induction on $\alpha$.

(1) Since $Y^{0}=Y=f(X)=f\left(X^{0}\right)$, then $Y^{\alpha} \subset f\left(X^{\alpha}\right)$ when $\alpha=0$.

(2) Suppose $Y^{\beta} \subset f\left(X^{\beta}\right)$ when $\beta<\alpha$. It suffices to show $Y^{\alpha} \subset f\left(X^{\alpha}\right)$ in the following two cases.

1) $\alpha=\beta+1$ for some ordinal number $\beta$.

Suppose $Y^{\alpha} \not \subset f\left(X^{\alpha}\right)$. Then $Y^{\alpha}-f\left(X^{\alpha}\right) \neq \emptyset$. Pick

$$
y \in Y^{\alpha}-f\left(X^{\alpha}\right) \text {. }
$$

Then $X^{\alpha} \cap f^{-1}(y)=\emptyset$. Put $F=X^{\beta}-f^{-1}(y)$.

Claim 1. $F$ is $*$-closed in $X$.

Put $A=X^{\beta} \cap f^{-1}(y)$. Then $F=X^{\beta}-A$. Since $X^{\alpha} \cap f^{-1}(y)=\emptyset$, $f^{-1}(y) \subset X-X^{\alpha}$. This implies $A \subset X^{\beta} \cap\left(X-X^{\alpha}\right)=X^{\beta}-X^{\alpha}$. By Remark 4.7, $X^{\beta}-X^{\alpha}=I^{*}\left(X^{\beta}\right)$. Thus $A \subset I^{*}\left(X^{\beta}\right)$. For any $x \in A, x \in I^{*}\left(X^{\beta}\right)$. Then $U \cap X^{\beta}=\{x\}$ for some $U \in \tau^{*}(x)$. Then $\{x\} \in \tau_{X^{\beta}}^{*}$ and so $A=\bigcup_{x \in A}\{x\} \in$ $\tau_{X^{\beta}}^{*}$. This implies $F=X^{\beta}-A \in \tau^{* \prime}{ }_{X^{\beta}}$. By Lemma 4.9(1), $F$ is $*$-closed in $X$.

By induction hypothesis, $Y^{\beta} \subset f\left(X^{\beta}\right)$. Then $Y^{\beta}-\{y\} \subset f\left(X^{\beta}\right)-\{y\}$. Note that $X^{\beta} \subset F \cup f^{-1}(y)$. Then $Y^{\beta}-\{y\} \subset f\left(F \cup f^{-1}(y)\right)-\{y\}=f(F)$. Thus $Y^{\beta}-f(F) \subset\{y\}$.

Conversely, by $f^{-1}(y) \cap F=\emptyset, y \notin f(F)$. Note that $y \in Y^{\alpha} \subset Y^{\beta}$. Then $\{y\} \subset Y^{\beta}-f(F)$.

Hence $Y^{\beta}-f(F)=\{y\}$.

Since $f$ is $*$-closed, by Claim $\mathbf{1}, f(F) \in \sigma^{* \prime}$. Note that $y \notin f(F)$. Put $U=Y-f(F)$. Then $U \in \sigma^{*}(y)$. By $U \cap Y^{\beta}=Y^{\beta}-f(F)=\{y\}, y \in I^{*}\left(Y^{\beta}\right)$. By Remark 4.7, $Y^{\beta}-Y^{\alpha}=I^{*}\left(Y^{\beta}\right)$. This implies $y \notin Y^{\alpha}$, a contradiction.

Therefore, $Y^{\alpha} \subset f\left(X^{\alpha}\right)$.

2) $\alpha$ is a limit ordinal number.

Suppose $Y^{\alpha} \not \subset f\left(X^{\alpha}\right)$. Then $Y^{\alpha}-f\left(X^{\alpha}\right) \neq \emptyset$. Pick

$$
y \in Y^{\alpha}-f\left(X^{\alpha}\right) \text {. }
$$

Put

$$
\pi=\max \left\{\beta: X^{\beta} \cap f^{-1}(y) \neq \emptyset\right\} .
$$


By condition $(*)$, we have $X^{\pi} \cap f^{-1}(y) \neq \emptyset$.

Since $X^{\alpha} \cap f^{-1}(y)=\emptyset$, we claim $\pi<\alpha$. Otherwise, we have $\pi \geqslant \alpha$. Since $X^{\pi} \cap f^{-1}(y) \neq \emptyset$ and $X^{\pi} \subset X^{\alpha}, X^{\alpha} \cap f^{-1}(y) \neq \emptyset$. Thus $y \in f\left(X^{\alpha}\right)$, a contradiction.

But $X^{\pi+1} \cap f^{-1}(y)=\emptyset$. Then $\{y\} \cap f\left(X^{\pi+1}\right)=\emptyset$ and so $f^{-1}(y) \cap$ $f^{-1}\left(f\left(X^{\pi+1}\right)\right)=\emptyset$.

Put

$$
W=X-f^{-1}\left(f\left(X^{\pi+1}\right)\right) .
$$

Then $f^{-1}(y) \subset W$. By Lemma $4.9(1), X^{\pi+1} \in \tau^{*^{\prime}}$. By $f$ is $*$-closed, $f\left(X^{\pi+1}\right) \in$ $\sigma^{*^{\prime}}$.

Put

$$
Z=Y-f\left(X^{\pi+1}\right) .
$$

Then $Z \in \sigma^{*}$ and $W=f^{-1}(Z)$. Put $g=\left.f\right|_{W}$.

Claim 2. $g: W \rightarrow Z$ is $*$-closed.

Let $K$ be $*$-closed in $W$. Then $K=F \cap W$ for some $F \in \tau^{* \prime}$. Since $f$ is *-closed, $f(F) \in \sigma^{* \prime}$. Note that

$$
g(K)=f(W \cap F)=f\left(f^{-1}(Z) \cap F\right)=Z \cap f(F) .
$$

Then $g(K)$ is *-closed in $Z$.

Since $X$ is $\mathcal{I}$-scattered, by Theorem $5.3, W$ is $\mathcal{I}_{W}$-scattered. By Theorem $4.11, \delta(W)$ is existence.

Claim 3. $\delta(W) \leqslant \pi+1$.

$W^{\pi+1} \subset W \subset X-X^{\pi+1}$. By Lemma $4.9(2), X^{\pi+1} \supset W^{\pi+1}$. Then $W^{\pi+1} \subset$ $X^{\pi+1} \cap\left(X-X^{\pi+1}\right)=\emptyset$. Thus $\delta(W) \leqslant \pi+1$.

Claim 4. $Y^{\alpha} \cap Z=Z^{\alpha}$.

(a) $\alpha=0$. We have $Z^{0}=Z=Y \cap Z=Y^{0} \cap Z$.

(b) Suppose $Y^{\beta} \cap Z=Z^{\beta}$ for any $\beta<\alpha$. We will prove $Y^{\alpha} \cap Z=Z^{\alpha}$ in the following cases.

a) $\alpha$ is a successor ordinal number.

By induction hypothesis, $Y^{\alpha-1} \cap Z=Z^{\alpha-1}$.

By $Y^{\alpha} \supset Z^{\alpha}$ and $Z \supset Z^{\alpha}$, we have $Y^{\alpha} \cap Z \supset Z^{\alpha}$.

Let $y \in Y^{\alpha} \cap Z$. By Remark 4.7, $Y^{\alpha}=Y^{\alpha-1} \cap d^{*}\left(Y^{\alpha-1}\right)$. Then $y \in$ $d^{*}\left(Y^{\alpha-1}\right) \cap Y^{\alpha-1} \cap Z=d^{*}\left(Y^{\alpha-1}\right) \cap Z^{\alpha-1}$. Note that $Z \in \sigma^{*}(y) . y \in d^{*}\left(Y^{\alpha-1}\right)$ implies $(U \cap Z) \cap\left(Y^{\alpha-1}-\{y\}\right) \neq \emptyset$ for any $U \in \sigma^{*}(y)$. Then

$$
\begin{aligned}
(U \cap Z) \cap\left(Y^{\alpha-1}-\{y\}\right) & =U \cap Z \cap Y^{\alpha-1} \cap\{y\}^{c} \\
& =U \cap Z^{\alpha-1} \cap\{y\}^{c}=U \cap\left(Z^{\alpha-1}-\{y\}\right) \neq \emptyset .
\end{aligned}
$$

Thus, $y \in d^{*}\left(Z^{\alpha-1}\right)$. By Remark 4.7,

$$
Z^{\alpha}=Z^{\alpha-1} \cap d^{*}\left(Z^{\alpha-1}\right) .
$$

Then $y \in Z^{\alpha}$. Hence $Y^{\alpha} \cap Z \subset Z^{\alpha}$.

Hence $Y^{\alpha} \cap Z=Z^{\alpha}$.

b) $\alpha$ is a limit ordinal number. 
By induction hypothesis, $Y^{\beta} \cap Z=Z^{\beta}$ for any $\beta<\alpha$. Then

$$
Y^{\alpha} \cap Z=\left(\bigcap_{\beta<\alpha} Y^{\beta}\right) \cap Z=\bigcap_{\beta<\alpha}\left(Y^{\beta} \cap Z\right)=\bigcap_{\beta<\alpha} Z^{\beta}=Z^{\alpha} .
$$

By Claim 2, $g: W \rightarrow Z$ is $*$-closed. Repeat the proof of 1$)$, we can prove $Z^{\pi+1} \subset g\left(W^{\pi+1}\right)$. By Claim 3, $\emptyset=W^{\delta(W)} \supset W^{\pi+1}$. This implies $Z^{\pi+1}=\emptyset$. By Remark 4.7(4), $Z=\bigcup_{\beta \leqslant \pi} I^{*}\left(Z^{\beta}\right)$.

Note that $X^{\pi+1} \cap f^{-1}(y)=\emptyset$. Then $y \notin f\left(X^{\pi+1}\right)$. So $y \in Z=\bigcup_{\beta \leqslant \pi} I^{*}\left(Z^{\beta}\right)$. We obtain $y \in I^{*}\left(Z^{\gamma}\right)$ for some $\gamma \leqslant \pi$. It follows $U \cap Z^{\gamma}=\{y\}$ for some $U \in \sigma^{*}(y)$.

By Claim 4, $Y^{\gamma} \cap Z=Z^{\gamma}$. Then $(U \cap Z) \cap Y^{\gamma}=U \cap Z^{\gamma}=\{y\}$. Since $U \cap Z \in \sigma^{*}(y)$, we have $y \in I^{*}\left(Y^{\gamma}\right)=Y^{\gamma}-Y^{\gamma+1}$. Then $y \notin Y^{\gamma+1}$.

Since $\pi<\alpha$ and $\alpha$ is a limit ordinal, $\pi+1<\alpha$. Then $\gamma+1 \leqslant \pi+1<\alpha$. By Remark 4.7, $Y^{\gamma+1} \supset Y^{\alpha}$. Then $y \notin Y^{\alpha}$, a contradiction.

Therefore, $Y^{\alpha} \subset f\left(X^{\alpha}\right)$.

Corollary 5.13. Let $(X, \tau, \mathcal{I})$ be $\mathcal{I}$-scattered, let $(Y, \sigma, \mathcal{J})$ be an ideal space and let $f:(X, \tau, \mathcal{I}) \rightarrow(Y, \sigma, \mathcal{J})$ be $*$-closed. If $\delta(X)$ is finite, then the following properties hold:

(i) $Y^{\alpha} \subset f\left(X^{\alpha}\right)$ for every ordinal number $\alpha$,

(ii) $\delta(Y) \leqslant \delta(X)$,

(iii) $Y$ is $\mathcal{J}$-scattered.

\section{References}

[1] G. Artico, U. Marconi, R. Moresco, and J. Pelant, Selectors and scattered spaces, Topology Appl. 111 (2001), no. 1-2, 105-134.

[2] H. R. Bennett and J. Chaber, Scattered spaces and the class MOBI, Proc. Amer. Math. Soc. 106 (1989), no. 1, 215-221.

[3] G. Bezhanishvili, R. Mines, and P. J. Morandi, Scattered, Hausdorff-reducible, and hereditarily irresolvable spaces, Topology Appl. 132 (2003), no. 3, 291-306.

[4] Z. Cai, D. Zheng, Z. Li, and H. Chen, $\mathcal{I}$-separability on ideal topological spaces, J. Adv. Res. Pure Math. 3 (2011), no. 4, 85-91.

[5] J. Dontchev, On Hausdorff spaces via topological ideals and $\mathcal{I}$-irresolute functions, Papers on general topology and applications (Slippery Rock, PA, 1993), 28-37, Ann. New York Acad. Sci., 767, New York Acad. Sci., New York, 1995.

[6] J. Dontchev, M. Ganster, and T. Noiri, Unified operation approach of generalized closed sets via topological ideals, Math. Japon. 49 (1999), no. 3, 395-401.

[7] J. Dontchev, M. Ganster, and D. Rose, Ideal resolvability, Topology Appl. 93 (1999), no. 1, 1-16.

[8] E. Ekici, On $\mathcal{I}$-Alexandroff and $\mathcal{I}_{g}$-Alexandroff ideal topological spaces, Filomat 25 (2011), no. 4, 99-108.

[9] S. Fujii, K. Miyazaki, and T. Nogura, Vietoris continuous selections on scattered spaces, J. Math. Soc. Japan. 54 (2002), no. 2, 273-281.

[10] E. Hatir, A. Keskin, and T. Noiri, A note on strong $\beta$-I-sets and strongly $\beta$-I-continuous functions, Acta Math. Hungar. 108 (2005), no. 1-2, 87-94.

[11] E. Hayashi, Topologies defined by local properties, Math. Ann. 156 (1964), 205-215.

[12] M. Henriksen, R. Raphael, and R. G. Woods, SP-scattered spaces; a new generalization of scattered spaces, Comment. Math. Univ. Carolin. 48 (2007), no. 3, 487-505. 
[13] D. S. Jankovic and T. R. Hamlett, New topologies from old via ideals, Amer. Math. Monthly 97 (1990), no. 4, 295-310.

[14] V. Kannan and M. Rajagopalan, Scattered spaces, Proc. Amer. Math. Soc. 43 (1974), $402-408$.

[15] _ Scattered spaces II, Illinois J. Math. 21 (1977), no. 4, 735-751.

[16] A. Keskin, T. Noiri, and S. Yuksel, Idealization of a decomposition theorem, Acta Math. Hungar. 102 (2004), no. 4, 269-277.

[17] K. Kuratowski, Topology, Academic Press, New York, 1966.

[18] Z. Li and F. Lin, On I-Baire spaces, Filomat 27 (2013), no. 2, 301-310.

[19] M. N. Mukherjee, B. Roy, and R. Sen, On extensions of topological spaces in terms of ideals, Topology Appl. 154 (2007), no. 18, 3167-3172.

[20] R. L. Newcomb, Topologies which are compact modulo an ideal, Ph.D. thesis, University of Cal. at Santa Barbara, 1967.

[21] V. Renuka Devi, D. Sivaraj, and T. Tamizh Chelvam, Codense and completely codense ideals, Acta Math. Hungar. 108 (2005), no. 3, 197-205.

[22] R. Vaidyanathaswamy, The localisation theory in set topology, Proc. Indian Acad. Sci. 20 (1944), 51-61.

ZHAOWEN LI

SCHOOL OF SCIENCE

Guangxi University for Nationalities Nanning, Guangxi 530006, P. R. China

E-mail address: lizhaowen8846@126.com

SHIZHAN LU

SCHOOL OF SCIENCE

Guangxi University for Nationalities

NAnning, Guangxi 530006, P. R. China

E-mail address: lubolin2006@163.com 